\title{
A Study on Pharmacist Mediated Intervention in Identifying and Reporting of Adverse Drug Reactions at a Tertiary Care Hospital
}

\section{Mohsina F.P ${ }^{1}$, Faheem I.P², Aquil-ur-Rahim Siddiuque ${ }^{3}$, Abhinandan Patil ${ }^{4}$, Mohammad A Kamal ${ }^{5,6,7}$, Mukim $\mathbf{M}^{8}$}

\author{
1,2Luqman College of Pharmacy, Gulbarga, Karnataka, India \\ ${ }^{3}$ Shri Bhagwan College of Pharmacy, Aurangabad, Maharashtra, India \\ ${ }^{4}$ Sanjay Ghodawat University, Kolhapur, Maharashtra, India \\ ${ }^{5}$ King Fahd Medical Research Center, King Abdulaziz University, P. O. Box 80216, Jeddah 21589, \\ Saudi Arabia \\ ${ }^{6}$ West China School of Nursing / Institutes for Systems Genetics, Frontiers Science Center for Disease-related \\ Molecular Network, West China Hospital, Sichuan University, Chengdu 610041, Sichuan, China \\ ${ }^{7}$ Enzymoics, 7 Peterlee Place, Hebersham, NSW 2770; Novel Global Community Educational Foundation, \\ Australia \\ ${ }^{8}$ Kota College of Pharmacy Kota, Kota Rajasthan, India.
}

Corresponding Author: Mohsina F.P

\begin{abstract}
Background: Adverse drug reactions are major problem in health care system due to its consequences - morbidity, mortality and health care cost. Recent epidemiologic research reveals that the total incidence rate of ADRs was 6.7\%, with an overall fatality rate of $0.32 \%$.

Method: A prospective observational study was carried out for 6 months in a tertiary care hospital. Patients admitted to the department of medicine, Pediatrics, orthopedics \& surgery were selected randomly and followed from date of admission to date of discharge by enrolling into the study by considering the study criteria.

Results: During the research about 27 ADRs were recognized \& reported. Among them 14 (51.86\%) patients were male and 13(48.14\%) were female patients. The system or organ most commonly involved was Blood disorders were 8 (29.62\%). Type A were 16(57.25\%) reactions followed by Type B were 8 (29.62\%); Type C were 3 (11.11\%). Study depicts drug class usually implicated with ADRs was Antibiotics and antineoplastic drugs were 6 (22.22\%) followed by Anti-Tubercular were 3 (11.11\%); NSAIDS and Antidiabetic drugs were 2 (7.4\%); The least implicated (3.7\%) drug class are AntiPyretic, Diuretics and Antiretroviral. Causality was assessed for all the reported reactions are
\end{abstract}

found to be PROBABLE according to WHOUMC Scale.

Conclusion: The study report shows that incidence of ADRs is steadily increasing. Under reporting of ADR is a major problem in India. Clinical Pharmacist plays a vital role in surveillance and prevention of ADRs.

Key Words: Adverse Drug Reactions, Pharmacovigilance, Causality.

\section{INTRODUCTION}

Adverse Drug Reaction [ADR]; According to WHO ADR is defined as any response to a drug which is noxious and unintended, and which occurs at doses normally used in man. ${ }^{1}$

Medication errors are commonly seen in hospitals. These can be further subdivided as prescribing error, dispensing error and administrative error. In whole, medication errors include wrong medication, wrong dose, wrong strength, wrong formulation, expired medication. ${ }^{2}$

The reports reveal that the total burden of thyroid disorder in India is 42 million. In India, Iodine deficiency disorders account for 27 per 1000 where as Grave's disease accounts 5 per 10,0002. The 
prevalence and pattern of Thyroid disorders depend on sex, age, ethnic and geographical factors and especially on iodine intake. Iodine deficiency can lead to mental retardation, still births, congenital anomalies and psychomotor defects. Research shows that hypothyroidism can contribute to morbidity from Osteoporosis, Hyperlipidemia, Hypercholesterolemia, Cardiovascular and Neuropsychiatry disease in the population. ${ }^{3}$ The seriousness of thyroid disorders should not be underestimated as thyroid storm and myxedema can lead to death in a significant number of cases. ${ }^{2}$

Drugs are vital in the management of diseases. A Drug related problem (DRP) is defined as an any event or circumstance involving drug treatment that interferes or potentially interferes with the patient achieving an optimum outcome of medical care. Cipolle classified drug related problems in to eight types.

1. Untreated indication

2. Use of medication without an indication

3. Improper drug selection.

4. Sub therapeutic dosage.

5. Over dosage.

6. Medication error/non-compliance

7. Drug interactions, and

8. Adverse drug reactions ${ }^{3}$

Based on the profile of medications prescribed, the drug-drug interactions are identified and classified.

1) Major: The effects are potentially life threatening or capable of causing permanent damage.

2) Moderate: The effects may cause deterioration in patients' clinical status and additional treatment or extension of hospital stay.

3) Minor: The effects are usually mild.

Drug interactions may lead to adverse drug reactions that can be severe enough to necessitate hospitalization and increased health care costs. About 5\% of all the adverse drug reactions in the hospitals are caused by Drug-Drug interaction, the majority of which are avoidable. Hence, this work is proposed to identify potential drug- drug interactions in the drug therapy by the clinical pharmacist and report the same to the physician so that adverse drug reactions can be prevented and patient outcome can be improved. ${ }^{4,5}$

As per WHO, Drug utilization studies and research information is the important part that helps in marketing, distribution and prescription pattern of drugs and helps to assess the impact of these on medical and socioeconomic status of patients. Thus, drug utilization studies are useful in understanding the prescription pattern along with the quality of prescription in terms of rationality, drug interactions and financial burden of disease to the individual. These information helps in improving the standards of treatment and identify the problems caused by polypharmacy, drugdrug interaction and adverse drug reactions. Periodically auditing of prescriptions in form of drug utilization studies are important tool to increase the efficacy of drug, to reduce the adverse effect, to optimize the cost of the treatment and to give better feedback to the medical practitioner. Previous studies conducted in Australia suggest that academic detailing improves the quality. ${ }^{6}$

The overuse and misuse of antibiotics has affected quality of life leading to larger side effects which caused financial burden to the patient, as well as over the health care system. Antibiotic use and antimicrobial resistance are increasing in India is reported by a "Community based Surveillance of Anti-microbial use and Resistance in the Resource constrained settings" by WHO based on the reports from 5 pilot projects three from India (Delhi, Mumbai, Vellore) and two from South Africa. ${ }^{7,8,9}$

It has been estimated that by 2050 , 700,000 deaths per year occur inevitably to antimicrobial resistance and, there might be 10 million deaths per year. ${ }^{10,11}$ Currently in India, even though most of medication errors remain unreported in many health care setups, we are still known to report 5.2 
million injuries related to medication errors and adverse drug reactions. ${ }^{12,13,14}$

Pharmaco-epidemiological research is described as the study to estimate the utilization pattern and effect of drugs in any clinical populations and to understand the various therapeutic outcomes like adverse drug reactions, drug effects like drug-drug interactions, medication adherence. It is the study of drug-oriented safety and its effectiveness. Rational usage of drugs is required for an effective treatment to achieve the therapeutic goals and maintain standards as per the established protocol. ${ }^{15}$

Atopic dermatitis (AD) is an inflammatory dermatological condition that extensively affects almost all parts of the body (upper extremities, lower extremities) with clear exemption of the groin and axillary regions. The pathogenesis of $\mathrm{AD}$ depends on environmental factors as a result there are wide variations in epidemiology from country to country. $\mathrm{AD}$ is a complex genetic disease with underlying epithelial barrier defect involving skin as well as mucosa hence is often accompanied by other atopic disorders such as allergic rhino conjunctivitis and asthma. Genetics, barrier dysfunction, defects in adaptive and immune response genes, immune dysregulation and microbial colonization are some of the mechanisms of pathogenesis. ${ }^{16,17}$

The most commonly observed antiepileptic drugs (AEDs) induced cutaneous adverse drug reactions (cADRs) include maculopapular exanthema (MPE), Stevens-Johnson Syndrome (SJS), Toxic Epidermal Necrolysis (TEN) and Drug Reaction with Eosinophilia and Systemic Symptoms (DRESS). ${ }^{1}$ There is an increased risk for Stevens-Johnson syndrome/toxic epidermal necrolysis (SJS/TEN) among HLA-B*15:02 positive patients and CBZ induced reactions such as Hypersensitivity Syndrome (HSS) whereas MPE occurs in Human Leukocyte antigen (HLA-A*31:01) positive patients and SJS/TEN and acute generalized exanthemata's pustulosis rarely occure. $^{18}$
Myasthenia gravis (MG) is an autoimmune disorder characterized by fatigue and muscular weakness. Around 15 percent of all cases of MG are graded as thymoma MG (T- MG). ${ }^{1,2}$ In the United States, the prevalence of T-MG is around 0.15 per 100,000 person- years. ${ }^{3,4}$ T-MG is categorized according to the histological classification of the World Health Organization (WHO) and graded according to the Masaoka staging system., ${ }^{5,6}$ The severity of this disorder is determined through the Myasthenia Gravis Foundation of America (MGFA) clinical classification system.7T-MG is popular on both men and women alike. It occurs at any age, with a 50-year peak start. Asians, Pacific Islanders and African Americans are more likely to contract this disease. When thymoma diagnosis is identified in an MG patient, the neoplasm should be surgically removed and radical excision of the neoplasm is crucial. Most T-MG appears to have difficult clinical development and poor prognosis. ${ }^{19}$

Localized infection of
Staphylococcus occurs in the skin, nose, mouth, throat, umbilicus and gastrointestinal tract (GIT). General malaise, irritability, fever, skin tenderness may be prominent. Other signs include facial edema, conjunctivitis and perioral crusting. ${ }^{20}$

Photosensitive eczema in combination with predominantly UVB sensitivity was identified as occurrence of eczema on photo exposed sites. Photo exposed site dermatitis and isolated UVA photosensitivity are also included in the concept of chronic actinic dermatitis in the absence of photoactive drugs, although much less common. Positive patch and/or photo patch results are found in the majority of identified patients. Photo testing and photo patch analysis facilities are not freely available and the histopathology is not comprehensive, the clinical characteristics remain the most important tool in chronic actinic dermatitis. The chronic actinic dermatitis treatment is generally divided into preventive, behavioral and environmental avoidance approaches to 
restrict photo-sensitivity clinical manifestations and suppressive methods to reduce the immune and inflammatory nature of the disease. ${ }^{21}$

The coronavirus outbreak has caused a major interruption to education activities in colleges and universities worldwide. The worsening pandemic threatens to affect certain aspects of college life, from admission, tuition, extra learning activities, boarding and other school activities. Since the WHO declared the coronavirus outbreak, the number of infections has burgeoned to worrying levels. For this reason, educational stakeholders decided on implementing school closure policies in order to slow down the rate of infection. Social distancing rules were also introduced to reduce close contact as a countermeasure to coronavirus infections. Social distancing rules were felt hard by pharmaceutical and other health care graduate students who were restricted from visiting healthcare facilities where they can observe and learn from experienced practitioners. The making of a competent pharmaceutical practitioner demands a hands-on practice and a physical presence which is majorly undermined by the coronavirus crisis. This paper seeks to explore the various effects coronavirus has on pharmaceutical education and the different counter measures taken to facilitate education. ${ }^{22}$

The science dealing with detecting, assessing, understanding and preventing ADRs has been termed "pharmacovigilance". Pharmacovigilance plays an essential role in the reduction of ADRs, thus the evolution and growth of this science are critical for effective and safe clinical practice. $^{23}$

As a result of thalidomide disaster, in 1968, the WHO started the Program for International Drug Monitoring (PIDM) for early detection of ADRs. This activity is now called as Pharmacovigilance. Ten members participated in this program (Australia, UK, USA, Germany, Canada, Ireland, Sweden, Denmark, New Zealand, and Netherlands). Italy participated in this program in $1975 .^{24}$

WHO promotes PV at the country level by working in collaboration with the Monitoring centre at Uppsala. More than 135 countries are the part of this program. Uppsala Monitoring Centre (UMC) in Sweden is responsible to monitor and manage the WHO-PIDM activities. This program not only enhances patient safety for use of medicines but also gives information about safe use and prevention and treatment of any Adverse Drug Reactions (ADRs). ${ }^{25}$

In 1992, the European Society of Pharmacovigilance (ESoP) was funded, turned into the International Society of Pharmacovigilance (IsoP). The aims of this society were to promote Pharmacovigilance, and enhance all aspects of the safe and proper use of medicines. ${ }^{26}$

The Pediatric Rule for Labelling, which was came in to action in1994 by the FDA and its 1998 enactment requiring manufacturers of certain new and marketed drugs to conduct studies for pediatric labelling has increased the information available regarding drug safety for children. ${ }^{27}$

India accounts for around $10 \%$ of global intake of medicines; the reporting of ADRs of medicines is a meagre $2 \%$ of the global occurrence. This is largely due to the poor reporting of adverse drug reactions in India. Despite this, India was 7thin position amongst the top ten countries contributing to global drug safety database. ${ }^{28}$

The ADR form is available at any pharmacovigilance center. The completed form should be sent to the peripheral pharmacovigilance center or in case of doubts, it can be sent directly to CDSCO. The information provided is handled in strict confidence. The peripheral center forwards the submitted form to the regional center where causality analysis is carried out, after MC. ${ }^{29}$

A cohort study assessed the ADR incidence rate in ambulatory patients as $25 \% .{ }^{4}$ ADR incidence has been reported to range from 5.9 to $22.3 \%$ of all emergency 
department admissions. ADRs are 4th-6th largest cause of death in USA.

A recent Swedish study has also implicated ADRs as 7th most common cause of death.5 The study of Bord et al indicated that, in patients who experience ADRs, death rates were $19.18 \%$ higher and the length of hospital stay is $8.25 \%$ higher. ${ }^{30}$

\section{General Objectives:}

A Study on Pharmacist Mediated Intervention in Identifying and Reporting Of Adverse Drug Reactions At A Tertiary Care Hospital

\section{Specific Objectives:}

$>$ To Assess Demographic Characteristics.

$>$ To Identify Types of Suspected ADRs.

$>$ To Identify Predisposing factor contributing the ADRs.

$>$ To Identify Drug Class and Medications involved in ADRs.

$>$ To Assess Disease Category by System Organ Class.

$>$ To Determine Seriousness of the ADRs.

$>$ To assess causality and severity of ADR's detected

$>$ To Determine the Outcome and Management of the suspected ADRs.

\section{METHODOLOGY}

STUDY SITE: Study was conducted at Tertiary Care Hospital.

SOURCE OF DATA: Case sheets of Inpatients admitted to the department of Medicine, Pediatrics, orthopedics and surgery at Tertiary Care Hospital.

STUDY DURATION: Study was carried out over a period of 6 months.

STUDY DESIGN: "A prospective observational study".

STUDY CRITERIA: Patients were enrolled into the study by considering study criteria.

\section{INCLUSION CRITERIA:}

- Patients who are willing to participate in the study.

- Patients of either Gender.
- Patients admitted to the department of medicine, paediatrics, surgery \& orthopaedics.

\section{EXCLUSION RITERIA:}

- Patients treated on out-patients basis were excluded from the study.

- Patients who were not willing to participate in the study.

\section{ETHICAL COMMITTEE APPROVAL:} The Human Ethical Clearance for the study was approved by Institutional Review Board (IRB).

\section{MATERIALS:}

The following study materials were prepared and used for the study. Informed Ascent/Consent Form

A Patient informed Ascent/Consent form was prepared and obtained from the patient before enrolling into the study by considering study criteria.

\section{Patient Data Collection Form}

A suitably designed Patient Data collection Form was prepared by referring standard textbooks, Journals and other relevant Sources which include information of Patient Demographic details such as Age, Gender, Duration of Disease, Hospital stay and Medication

\section{History}

- Yellow Card

- Red Alert Card

- Casualty Assessment Scale

\section{STUDY PROCEDURE:}

A Prospective observational study was carried out for a period of 6 months in a Tertiary care hospital. Patients admitted at Tertiary care hospital were selected randomly and enrolled into the study by considering the study criteria. Patient Informed Ascent/Consent proforma was taken from each patient at the time of enrollment into the study. Details regarding demography, laboratory investigations, past medical and medication history and current medication history was collected from the case sheets and noted in the suitably design 
patient data collection proforma. The Patients who developed ADR were identified and reported. ADR identified was analyzed using causality assessment scales Naranjo scale, WHO scale and Hartwig Severity Scale.

\section{RESULT}

In our study 27 suspected ADRs were identified and reported among 50 patients during six-month prospective observational study period. Accounting for an incidence of 32.05\%. 24 (41.3\%) patients were hospitalized due to ADRs, whereas 34 (58.6\%) patients developed ADRs during their hospital stay. A maximum of 2 ADRs were reported among 12 patients (20.68\%). It was observed that average length of the hospital stays for patients who developed ADRs was higher than patients without ADR.

Reported ADRs were evaluated for the following Parameters:

- Demographics - Age and Gender.

- Type of ADR reaction Mechanism.
- Predisposing factors.

- Drug class involved in ADRs.

- Individual Drug implicated.

- System Organ Effected.

- Frequency of ADRs in effected Organ.

- Characteristics of ADRs.

- Seriousness of the ADRs.

- Duration of the ADRs.

- Causality Assessment.

- Naranjo scale.

- WHO-UMC Scale.

- Hartwig severity scale.

- Outcomes of ADRs.

- Management of ADRs.

\section{Demographic Characteristics of Patients:}

Table 1: Details on Gender Distribution among the Patients with ADR in the study

\begin{tabular}{|l|l|l|l|}
\hline S. No. & Description & Total & Percentage \\
\hline 1. & Male & 14 & $51.86 \%$ \\
\hline 2. & Female & 13 & $48.14 \%$ \\
\hline
\end{tabular}

During the study period, 27 patients were analysed, among them 14(51.86\%) patients were male and 13 (48.14\%) were female patients.

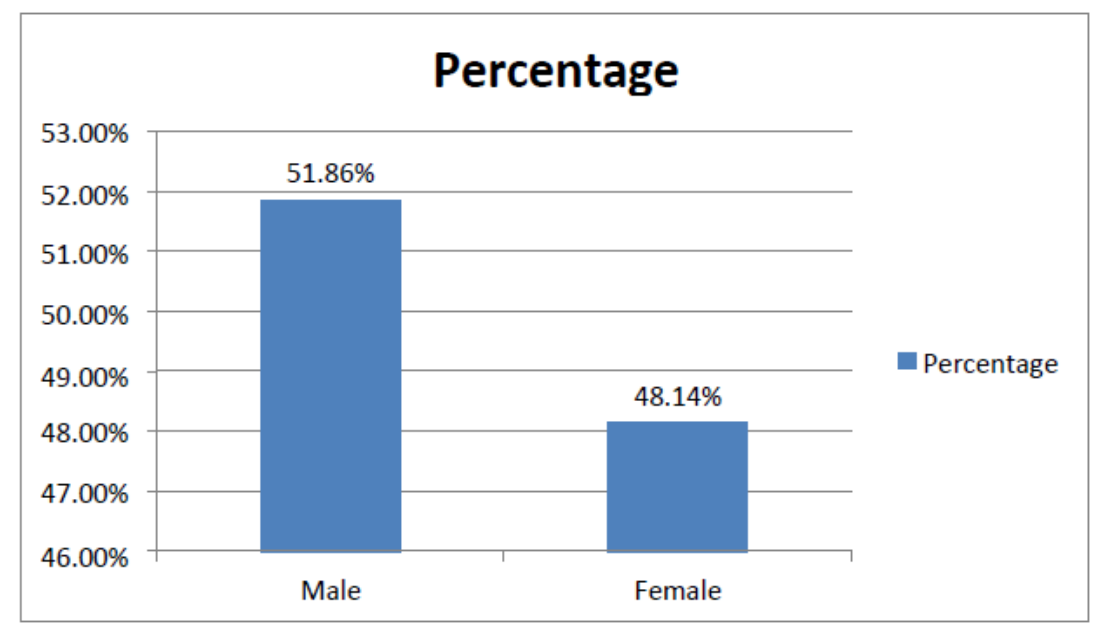

Figure 1: Details on Gender Distribution among the Patients with ADR in the study.

The Incidence of ADRs is higher in Males (50\%) than in Females (50\%).

Table 2: Details on age wise distribution among the patients with ADR in the study

\begin{tabular}{|l|l|l|}
\hline Age & No. of ADRs & \% ADRs \\
\hline $11-20$ & 00 & $00 \%$ \\
\hline $21-30$ & 08 & $29.62 \%$ \\
\hline $31-40$ & 03 & $11.11 \%$ \\
\hline $41-50$ & 08 & $29.62 \%$ \\
\hline $51-60$ & 03 & $11.11 \%$ \\
\hline $61-70$ & 03 & $11.11 \%$ \\
\hline $71-80$ & 02 & $7.40 \%$ \\
\hline Total & 27 & $100 \%$ \\
\hline
\end{tabular}

In a total of 27 Patients, $00(00 \%)$ ADRs were found in the age group between 11-20 years followed by 08 (29.62\%) ADRs were found in the age group between 21-30 years, 03 (11.11\%) ADRs were found in the age group between 31-40years, 08(29.62\%) ADRs were found in the age group between 41-50years, 03(11.11\%) ADRs were found in the age group between 51-60 years, 03(11.11\%) ADRs were found in the age 
Mohsina F.P et.al. A study on pharmacist mediated intervention in identifying and reporting of adverse drug reactions at a tertiary care hospital.

group between 61-70 years and 02(07.40\%) Years.

ADRs were found in the age group of 71-80

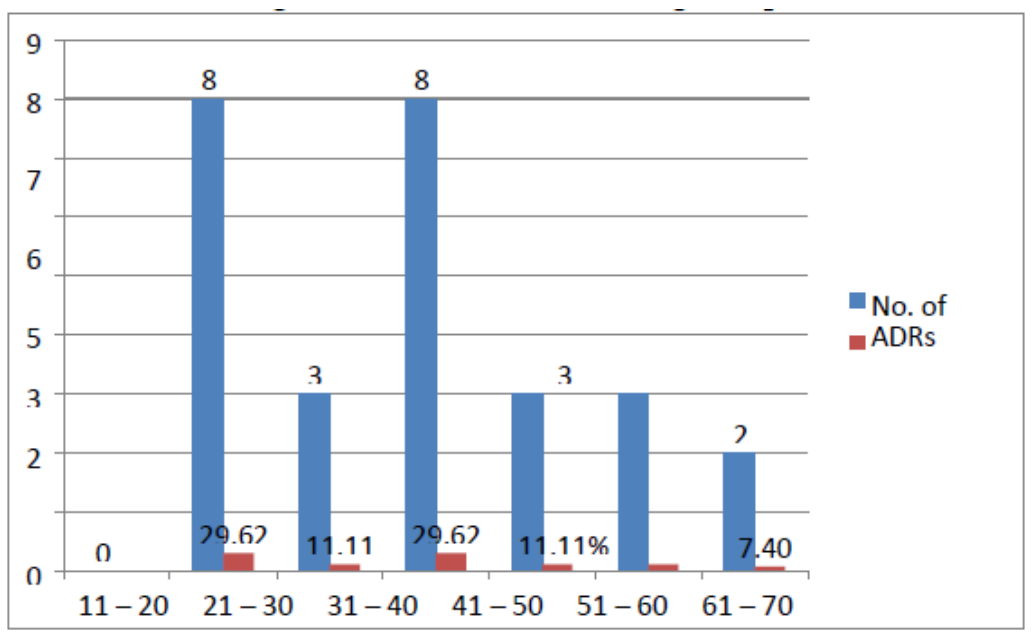

Figure 2: Details on age wise distribution among the patients with ADR in the study.

Table 3: Types of suspected ADR Reaction Mechanism

\begin{tabular}{|l|l|l|}
\hline ADR Type & No. of ADR & Percentage \\
\hline Type A & 16 & $59.25 \%$ \\
\hline Type B & 08 & $29.62 \%$ \\
\hline Type C & 03 & $11.11 \%$ \\
\hline Type D & 00 & $0 \%$ \\
\hline Type E & 00 & $0 \%$ \\
\hline Type F & 00 & $0 \%$ \\
\hline Type G & 00 & $0 \%$ \\
\hline Type H & 00 & $0 \%$ \\
\hline Type U & 00 & $0 \%$ \\
\hline
\end{tabular}

Most (59.25\%) of the reactions were Type A reactions followed by Type B (29.62\%); Type C (11.11\%). None of the reported ADR belonged to Type D, E, F, G, $\mathrm{H}, \mathrm{U}$.

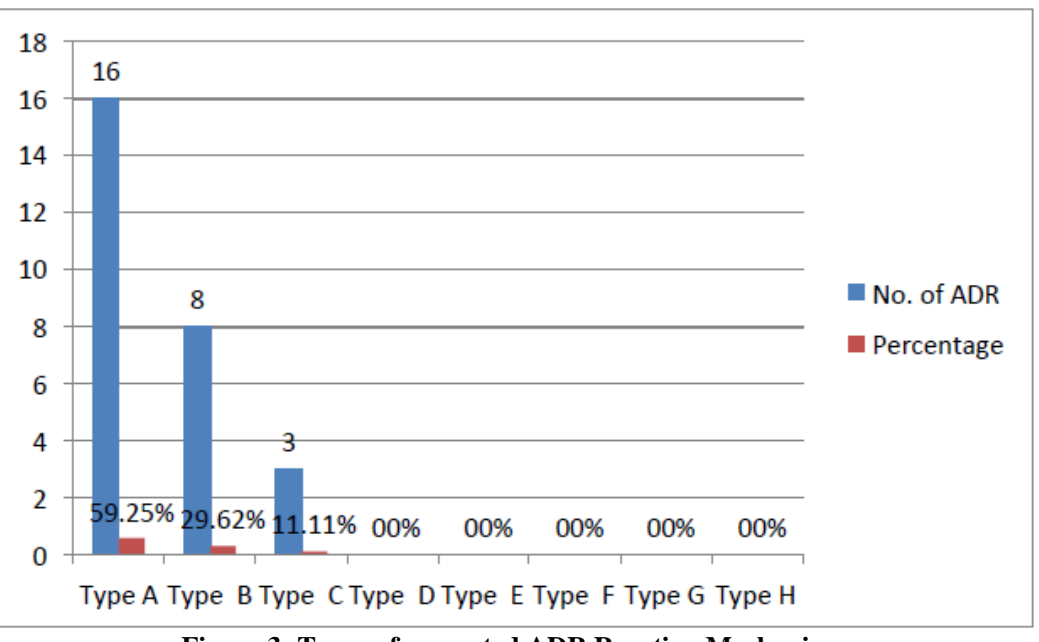

Figure 3: Types of suspected ADR Reaction Mechanism.

The Incidence of Type A ADR is higher than other types.

Table 4: Predisposing Factors.

\begin{tabular}{|l|l|l|}
\hline Parameter & No. of ADRs & Percentage \\
\hline Multi Drug Therapy & 05 & $18.51 \%$ \\
\hline Intercurrent Diseases & 17 & $62.96 \%$ \\
\hline Age (>60years) & 05 & $18.51 \%$ \\
\hline
\end{tabular}

Most of the patients who developed ADRs were on multi drug therapy
(Polypharmacy) and was the highest predisposing factors for most of the ADRs (05). Second predisposing factor of ADRs responsible was Age, 05 (18.51\%) reactions were reported with age more than 60 years. Out of 27 ADRs 17(62.96\%) reactions were due to intercurrent disease. The predisposing factors of the reported ADRs are 
Mohsina F.P et.al. A study on pharmacist mediated intervention in identifying and reporting of adverse drug reactions at a tertiary care hospital.

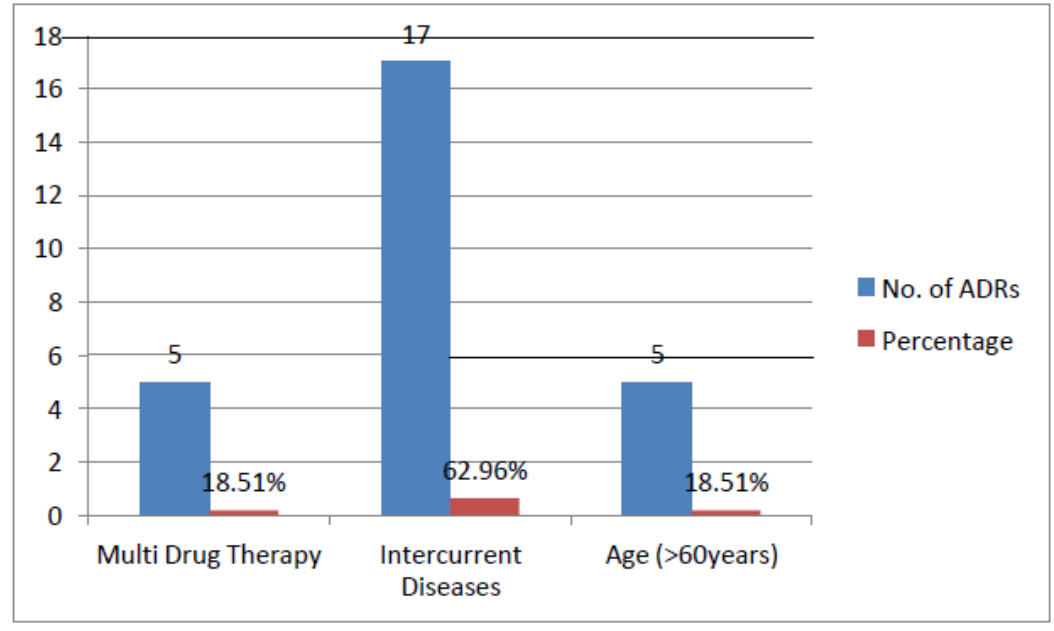

Figure 4: Predisposing Factors.

Table 5: Drug Class involved in ADRs

\begin{tabular}{|l|l|l|}
\hline Drug Class & Number of ADRs & Percentage \% \\
\hline Anti-retroviral & 01 & $3.7 \%$ \\
\hline Antipyretic & 01 & $3.7 \%$ \\
\hline Antitubercular & 03 & $11.11 \%$ \\
\hline Diuretics & 01 & $3.7 \%$ \\
\hline Antineoplastic & 06 & $22.22 \%$ \\
\hline Antiepileptic & 01 & $3.7 \%$ \\
\hline NSAIDS & 02 & $7.4 \%$ \\
\hline Anti-biotics & 06 & $22.22 \%$ \\
\hline Anti-diabetics & 02 & $7.4 \%$ \\
\hline Anti enemia & 01 & $3.7 \%$ \\
\hline Anti-rheumatoid & 01 & $3.7 \%$ \\
\hline Anti-metabolite & 01 & $3.7 \%$ \\
\hline Anti-Coagulants & 01 & $3.7 \%$ \\
\hline
\end{tabular}
implicated with ADRs was Antibiotics (16.66\%) followed by NSAIDS (13.33\%); Anti-Tubercular (10\%); Antiretroviral, Anti-Convulsant, AntiCoagulant and Anti-Psychotic are having equal Predominance (6.66\%); The least implicated (3.33\%) drug class are AntiPyretic, Diuretics, Anti-anginal, Multivitamins, Anti-Diabetics, Corticosteroids, Anti-Malarial, AntiParkinsonism, Analgesic and Iron supplements.

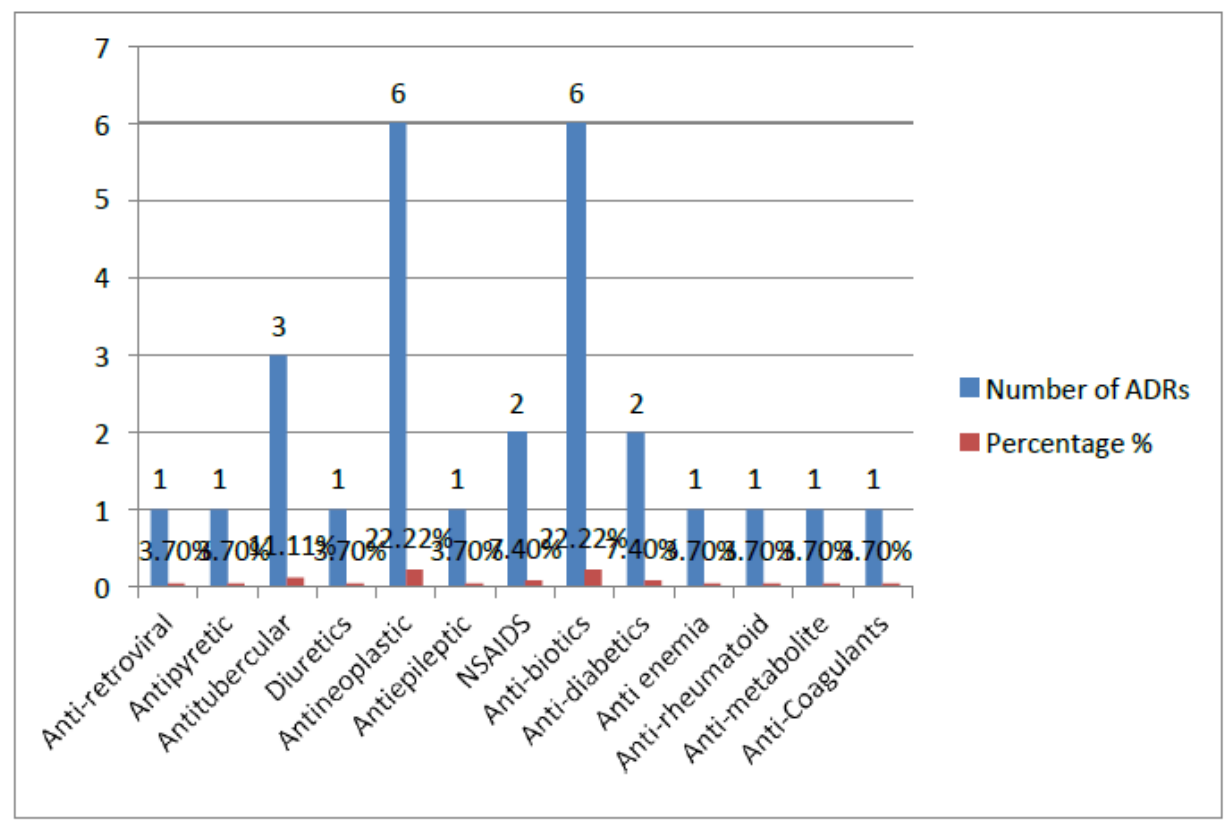

Figure 5: Drug Class Involved in ADRs.

The higher incidence of ADRs is associated with Antibiotics.

The least incidence of ADRs is associated with Anti-parkinsonism, Anti - Malarial and Anti-psychotics. 
Mohsina F.P et.al. A study on pharmacist mediated intervention in identifying and reporting of adverse drug reactions at a tertiary care hospital.

Table 6: Drugs Implicated in ADRs.

\begin{tabular}{|l|c|c|c|}
\hline Table 6: Drugs Implicated in ADRs. \\
\hline Individual Drug & Route & No. of ADRs & \% of ADRs \\
\hline Metformin 500mg & PO & 01 & $3.7 \%$ \\
\hline Methotrexate 2.5mg & PO & 01 & $3.7 \%$ \\
\hline Sodium valproate & PO & 01 & $3.7 \%$ \\
\hline Amikacin & PO & 01 & $3.7 \%$ \\
\hline Paracetamol 500mg & IV & 01 & $3.7 \%$ \\
\hline Cefotaxime 1mg & IV & 01 & $3.7 \%$ \\
\hline AkT4 & PO & 01 & $3.7 \%$ \\
\hline Zidovudine & PO & 01 & $3.7 \%$ \\
\hline Carboplatin & PO & 01 & $3.7 \%$ \\
\hline Vincristine 2mg & PO & 01 & $3.7 \%$ \\
\hline Enoxaparin 100mg & PO & 01 & $3.7 \%$ \\
\hline AKT4+Forcox & PO & 01 & $3.7 \%$ \\
\hline Diclofenac 50mg+Paracetamol 325mg & PO & 01 & $3.7 \%$ \\
\hline Docetaxel 100mg & PO & 01 & $3.7 \%$ \\
\hline Gemcitabine 1.49mg & PO & 01 & $3.7 \%$ \\
\hline Rituximab & PO & 01 & $3.7 \%$ \\
\hline Docetaxel 60mg + leulovorin 300mg & IV & 01 & $3.7 \%$ \\
\hline Forcox & PO & 01 & $3.7 \%$ \\
\hline Lasix 20mg & PO & 01 & $3.7 \%$ \\
\hline Sulphamethoxazol & PO & 01 & $3.7 \%$ \\
\hline Paclitaxel & PO & 01 & $3.7 \%$ \\
\hline Mitomycin & IV & 01 & $3.7 \%$ \\
\hline Doxorubicin & IV & 01 & $3.7 \%$ \\
\hline Vintor 4000IV (Erythopectin) & IV & 01 & $3.7 \%$ \\
\hline OHA(Glemperide 2mg+Metformin500mg) & PO & 01 & $3.7 \%$ \\
\hline Ornindazole 200mg+Ofloxacin500mg & PO & 01 & $3.7 \%$ \\
\hline Etoricoxib 90mg & IV & 01 & \\
\hline & & & \\
\hline
\end{tabular}

The drugs most commonly implicated in ADR were AKT4+ Forcox (11.11\%), Metformin, Paracetamol, Diclofenac, Docetaxel, (7.4\%), Methotrexate, sodium valproate, Amokacine, Cefotaxime, Zidovudine, Carboplatine, Vincristine, Enaxoprine, Gemicita bine, Rituximab, Lasix, Sulphamethaxazol, Paclitaxel, Mitomycine, Doxorubicin, Vintor, Ornindazole, Etoxicoxib (3.7).

Most commonly affected System Organ in the study was Blood (29.62\%), followed by Other (22.22\%) then Systems like renal (18.51\%). Skin (11.11\%). Hepatic and CNS (7.4\%) Gastrointestinal (3.7\%) were also found to be affected due to ADRs are least effected.

Table 7: System most commonly associated with ADRs

\begin{tabular}{|l|l|l|}
\hline System Involved In ADR & No. of ADRs & Percentage \% \\
\hline Skin & 03 & $11.11 \%$ \\
\hline Renal & 05 & $18.51 \%$ \\
\hline Hepatic & 02 & $7.40 \%$ \\
\hline Gastrointestinal & 01 & $3.7 \%$ \\
\hline CNS & 02 & $7.40 \%$ \\
\hline Blood & 08 & $29.62 \%$ \\
\hline Others & 06 & $22.22 \%$ \\
\hline
\end{tabular}

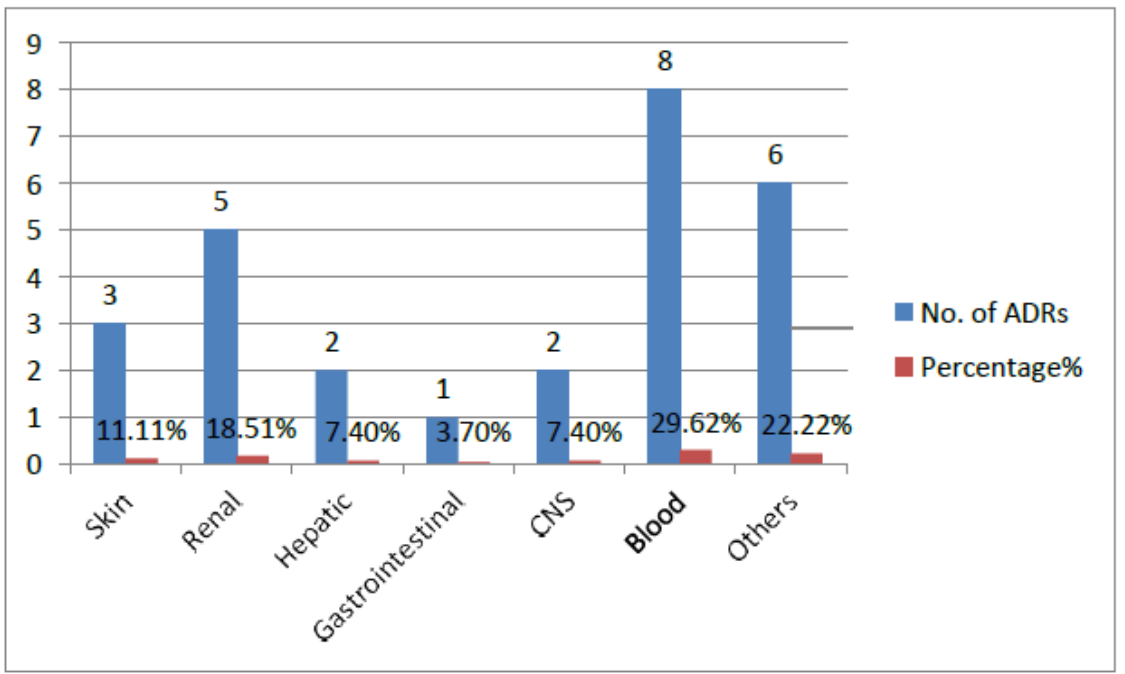

Figure 6: System most commonly associated with ADRs 
Mohsina F.P et.al. A study on pharmacist mediated intervention in identifying and reporting of adverse drug reactions at a tertiary care hospital.

The higher incidence of ADRs was and Renal system having equal seen in skin (20.78\%), following by Blood Predominance (14.28\%).

Table 8: Reported Reactions of System Organ Class.

\begin{tabular}{|c|l|c|}
\hline Organ System & Symptoms/Complaint & Total Number \\
\hline Skin & Steven Johnson Syndrome, & $02(7.4 \%)$ \\
& Blister on hand & $01(3.7 \%)$ \\
\hline Gastrointestinal & Gastritis & $01(3.7 \%)$ \\
\hline Blood & Anemia, Leukopenia, & $01(3.7 \%)$ \\
& Thrombocytopenia, Hemorrhage, & $01(3.7 \%)$ \\
& Decreased platelet count, & $02(7.4 \%)$ \\
& Thrombocytopenia Anemia & $01(3.7 \%)$ \\
& Febrile neutropenia & $01(3.7 \%)$ \\
& & $02(7.4 \%)$ \\
\hline Renal & Increase creatinine level & $01(3.7 \%)$ \\
& Urinary tract infection, & $01(3.7 \%)$ \\
& Nephropathy, Nephrotoxicity, & $01(3.7 \%)$ \\
& Grade IV chronic kidney disease & $01(3.7 \%)$ \\
\hline Hepatic & & $01(3.7 \%)$ \\
\hline CNS & Hepatitis & $02(7.4 \%)$ \\
\hline Others & Neuropathy & $02(7.4 \%)$ \\
& Infusion Reaction & $01(3.7 \%)$ \\
& Hypokalemia & $01(3.7 \%)$ \\
& Hypoglycemia, & $02(7.4 \%)$ \\
& Hyponatremia & $01(3.7 \%)$ \\
\hline
\end{tabular}

In the Current study, majority of the suspected reactions were Steven Johnson's syndrome, Febrile Neutropenia, Hemorrhage, Hepatitis, Neuropathy, Hypoglycemia (7.4\%) which are fatal to the patients followed by Blister on hand, Grade IV chronic kidney disease, gastritis, Anemia, Leukopenia, Decreased platelets count, Thrombocytopenia Anemia, Increased creatinine level, UTI, Nephropathy, Nephrotoxicity, Infusion reaction, Hypokalemia, Hyponatremia, Hypoglycemic attack (3.7\%).

Table 9: Seriousness of the Reaction.

\begin{tabular}{|l|l|l|}
\hline Parameters & No. of ADRs & Percentage \\
\hline Serious & 04 & $7.40 \%$ \\
\hline Life Threatening & 04 & $7.40 \%$ \\
\hline Death & 00 & 00 \\
\hline Non-serious & 23 & $42.59 \%$ \\
\hline Required Intervention & 20 & $37.03 \%$ \\
\hline Hospitalization & 03 & $5.55 \%$ \\
\hline
\end{tabular}

Among 30 ADRs, 13(43.33\%) ADRs are found to be Non-serious and 07(23.33\%) ADRs are noticed as Serious. 07 serious reactions are found to be Life Threatening. None of the reported reactions led to death. Out of 13 non-serious reactions 1 is found to be hospitalized. Seriousness of the Reactions

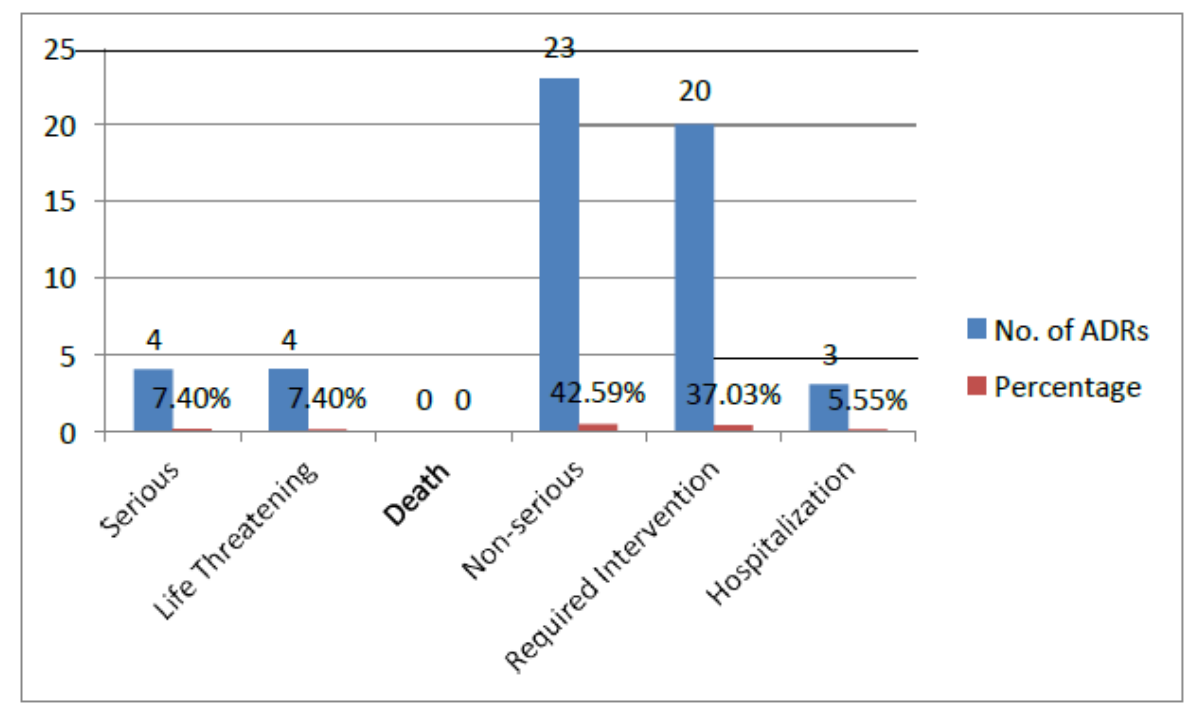

Figure 7: Seriousness of the Reaction. 
Mohsina F.P et.al. A study on pharmacist mediated intervention in identifying and reporting of adverse drug reactions at a tertiary care hospital.

Table 10: Duration of the Reaction.

\begin{tabular}{|c|c|c|}
\hline Parameter & No. of ADRs & Percentage \\
\hline$<1$ day & 03 & $11.11 \%$ \\
\hline $1-5$ days & 20 & $74.07 \%$ \\
\hline$>$ 5days & 04 & $14.81 \%$ \\
\hline
\end{tabular}

Majority of the ADRs (74.07\%) are cured within 1-5 days, whereas 03(11.11\%) ADRs took less than one day to cure and 04(14.81\%) ADRs took more than 5 days to cure.

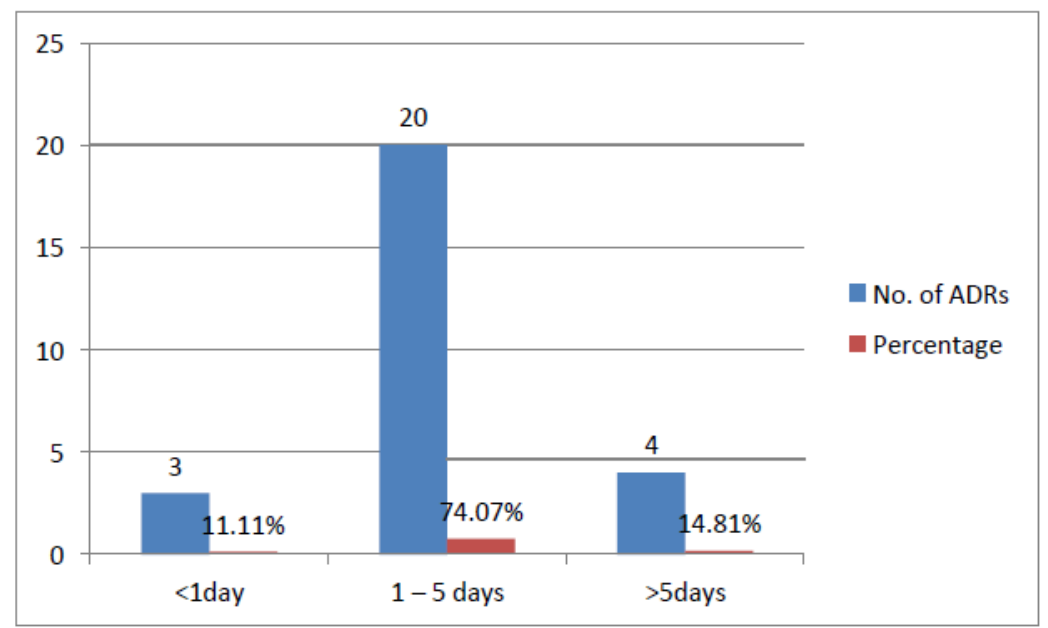

Figure 8: Duration of the Reaction.

\section{Causality Assessment}

Table 11: Naranjo Scale.

\begin{tabular}{|l|l|l|}
\hline Parameter & No. of ADRs & Percentage \\
\hline Certain/Definite & 00 & 00 \\
\hline Probable & 03 & $11.11 \%$ \\
\hline Possible & 24 & $88.88 \%$ \\
\hline Unlikely & 00 & 00 \\
\hline
\end{tabular}

According to Naranjo Scale, 24(88.88\%) reactions are found to be Possible and only 03(11.11\%) are found to be Probable.

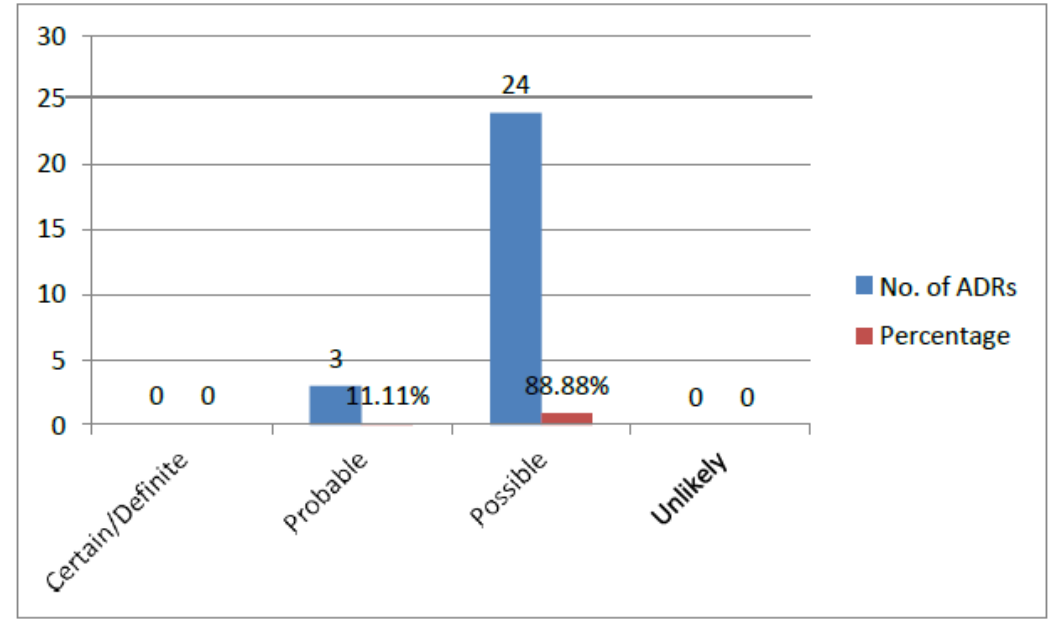

Figure 9: Naranjo Scale.

Table 12: WHO-UMC Scale.

\begin{tabular}{|l|l|l|}
\hline Parameter & No. of ADRs & Percentage \\
\hline Certain & 00 & $0 \%$ \\
\hline Probable/Likely & 22 & $81.48 \%$ \\
\hline Possible & 05 & $18.51 \%$ \\
\hline
\end{tabular}


Mohsina F.P et.al. A study on pharmacist mediated intervention in identifying and reporting of adverse drug reactions at a tertiary care hospital.

According to WHO scale, 22(81.48\%) reactions are Probable/Likely and remaining 05(18.51\%) reactions are possible.

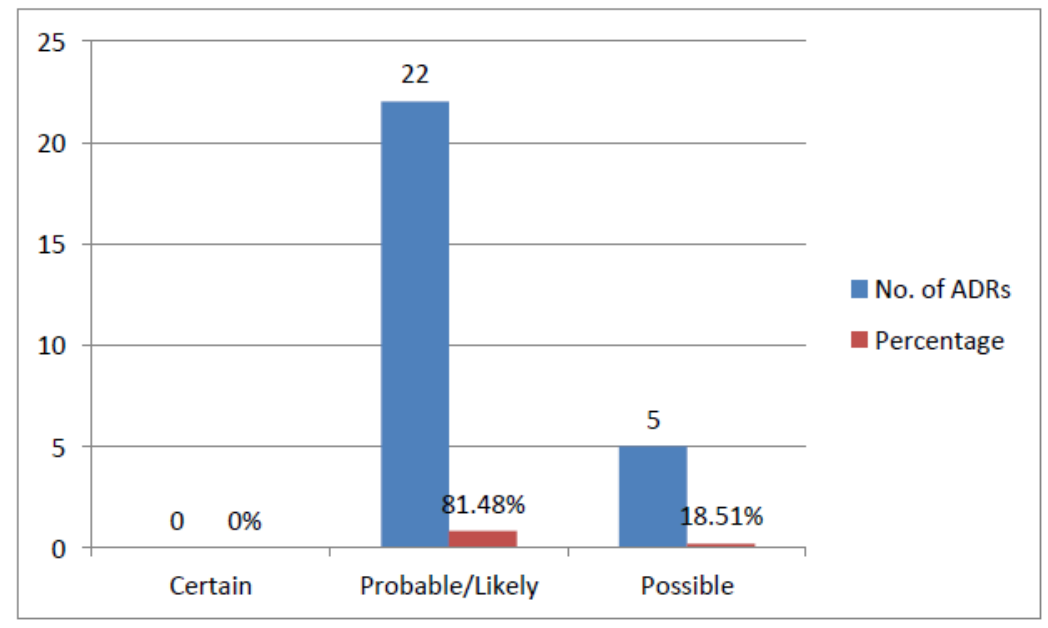

Figure 10: WHO-UMC Scale.

Table 13: Hartwig Severity Scale.

\begin{tabular}{|l|l|l|}
\hline Parameter & No. of ADRs & Percentage \\
\hline Mild & 07 & $25.92 \%$ \\
\hline Moderate & 17 & $62.96 \%$ \\
\hline Severe & 03 & $11.11 \%$ \\
\hline
\end{tabular}

According to Hartwig Severity Scale, 17(62.96\%) ADRs are moderate, 07(25.95\%) ADRs are mild, 03(11.11\%) ADRs are Severe.

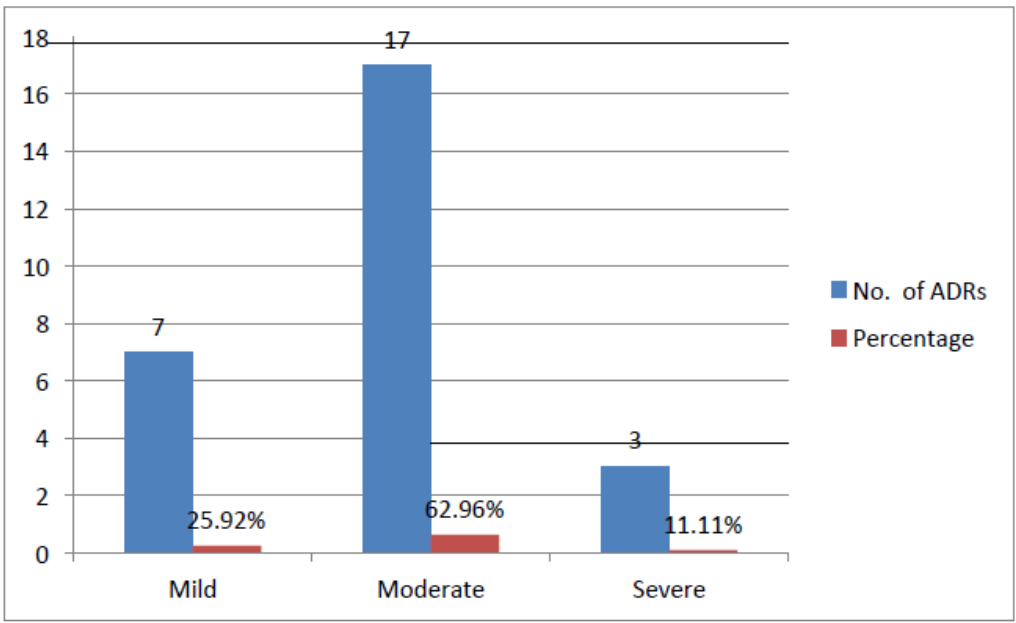

Figure 11: Hartwig Severity Scale.

Table 14: Outcomes of ADRs.

\begin{tabular}{|l|l|l|}
\hline Parameter & No. of ADRs & Percentage \\
\hline Unknown & 01 & $3.7 \%$ \\
\hline No recovery & 02 & $7.4 \%$ \\
\hline Recurrent of symptoms & 04 & $14.81 \%$ \\
\hline Recovered & 17 & $62.96 \%$ \\
\hline Continuing & 02 & $7.4 \%$ \\
\hline Fatal & 01 & $3.7 \%$ \\
\hline
\end{tabular}

Among 27 ADRs, 17(62.96\%) ADRs are recovered, 02 (7.4\%) ADRs are Unrecovered, 02(7.4\%) are remained as Continuing,01(3.7\%) are unknown,01(3.7\%) are fatal,04(14.81\%). 
Mohsina F.P et.al. A study on pharmacist mediated intervention in identifying and reporting of adverse drug reactions at a tertiary care hospital.

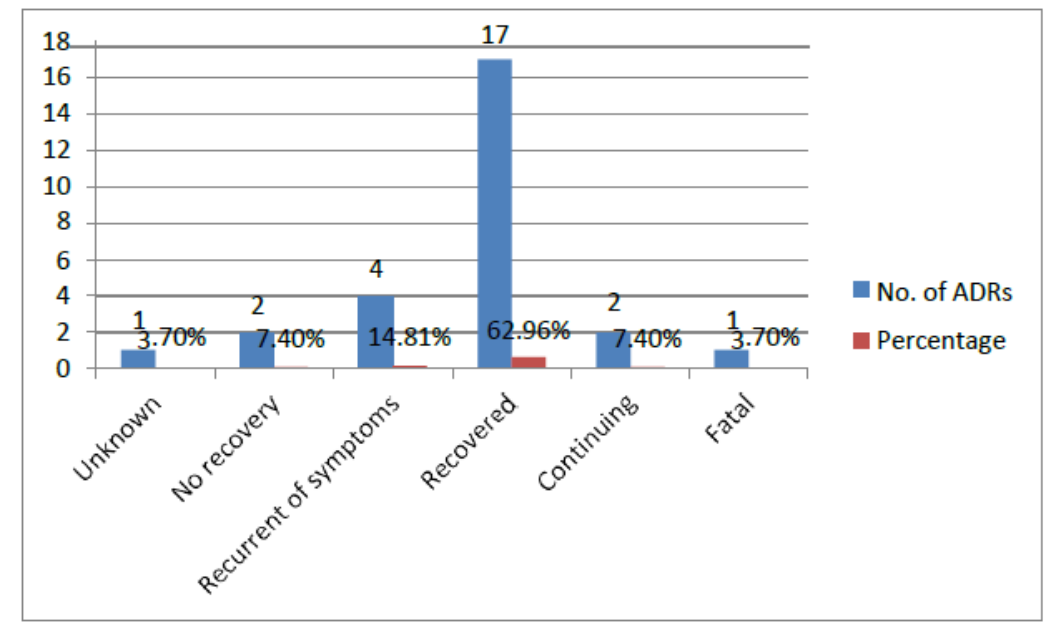

Figure 12: Outcomes of ADRs.

Table 15: Management of ADRs

\begin{tabular}{|l|l|l|}
\hline Parameter & No. of ADRs & Percentage \\
\hline Added Another Drug & 00 & $0 \%$ \\
\hline Stopped the Medication & 19 & $70.37 \%$ \\
\hline Substituted another Drug & 05 & $18.51 \%$ \\
\hline No Change & 00 & $0 \%$ \\
\hline Reduced Dose & 03 & $11.11 \%$ \\
\hline Reintroduction & 00 & $0 \%$ \\
\hline No Information & 00 & $0 \%$ \\
\hline
\end{tabular}

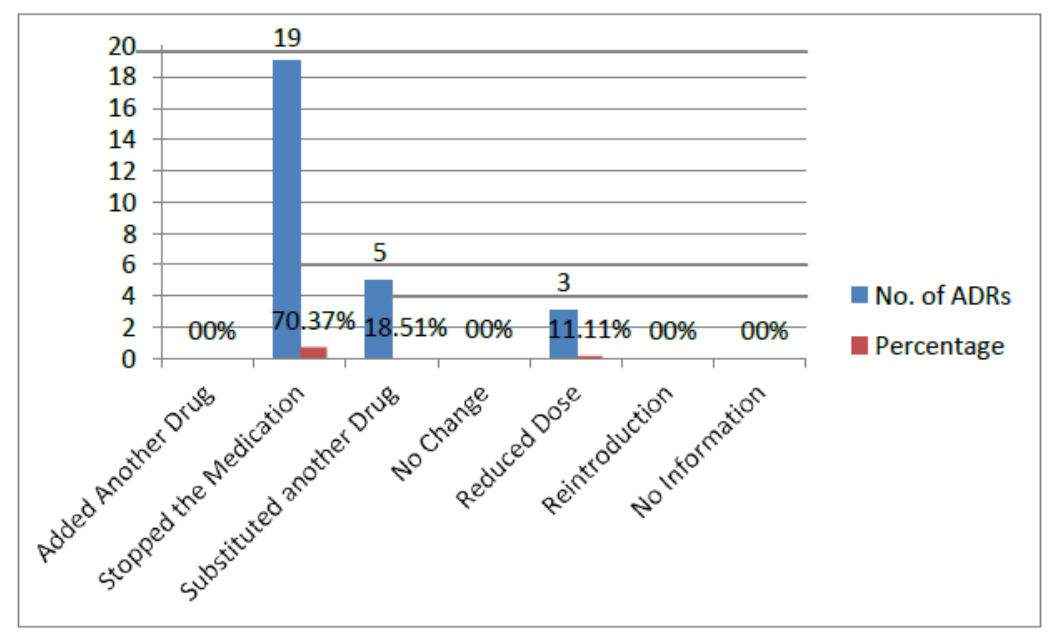

Figure 13: Management of ADRs.

\section{DISCUSSION}

In the pharmacotherapy of various diseases, most of the drugs are likely to have a dual effect- beneficial as well as adverse effect. So, the best way to control these adverse effects is to have a triple pronged approach of prevention, treatment and rehabilitation. Globally the incidences of adverse events were around 43 million each year and caused disability among 23 million. Among this two third occur in low income and middle-income countries. Adverse Drug Reactions were intensively monitored, documented and analyzed by
Causality Assessment Scale. ADRs were evaluated by considering the Clinical investigations, Drug Dosing and Frequency. It was observed that average length of the hospital stay for patients who developed ADRs was higher than patients without ADR. Gender distribution of the patients showed that there were $14(51.86 \%)$ male and 13(48.14\%) female Patients indicating male Preponderance. Age Distribution of the Patients showed that majority (29.62.\%) of ADRs was seen in the Age group 21- 30 \& 41-50years. Adult predominance $(51.87 \%)$ is more than the pediatric $(0 \%)$ 
and geriatric (18.51\%) patients. In the present study, the most common type of ADR is Type A with an incidence of 59.25\%. As Type a Reactions are dose related in the development of ADR and can be almost preventable. Incidence of Type B is $29.62 \%$; Type $\mathrm{C}$ is $11.11 \%$. None of the reported reactions included in Type $\mathrm{D}, \mathrm{E}, \mathrm{F}$, $\mathrm{G}, \mathrm{H}$ and $\mathrm{U}$. In our study, most of the patients who developed ADRs were on Intercurrent Disease 17(62.96\%) and was the highest predisposing factors for most of the ADRs. Second predisposing factor of ADRs responsible was Age, 05 (18.51\%) reactions were reported with age more than 60 years. Out of 27 ADRs only 5 (18.51\%) reactions were due to Multi Drug Therapy. The study shows the drug class most commonly implicated with ADRs was Antibiotics \& Antineoplastic (22.22\%) followed by Antitubercular (11.11\%); NSAIDS \& Antidiabetic (7.4\%); Antiretroviral, Anti-Coagulant, Anti pyretic, Diuretics, Anti-Epileptic, Anti-Anemia, Anti Rheumatoid, Anti Metabolite (3.7\%). The drugs most commonly implicated in ADR were Amlodipine (6.45\%), Diclofenac (5.17\%), Combination Antitubercular drugs (5.47\%), and Combination Anti-diabetic drugs (3.87\%). Other Drugs like Carbamazepine (3.87\%), Orofer, Phenytoin, has equal predominance $(2.57 \%)$, Sodium Valproate, Cefuroxime, Cefotaxime, Ceftriaxone, Mitomycin, Omnocortisol and Fentanyl have an equal Predominance (1.27\%). Most commonly affected System Organ in the study was blood (29.62\%), followed by others (22.22\%) then Systems like Renal (18.51\%). Skin (11.11\%). Hepatic and CNS (7.4\%). and Gastrointestinal (3.7) were also found to be affected due to ADRs are least effected. In the Current study, majority of the suspected reactions were Anemia (11.11\%) which are fatal to the patients followed by Steven Johnson Syndrome, Hepatitis, Neuropathy, Hemorrhage, Hypoglycemia, Febrile Neutropenia (7.4\%) Blister on hand, Gastritis, Decreased platelet count, Increased creatinine level, Urinary tract infection, Nephropathy, Nephrotoxicity, Grad IV, chronic Kidney disease, Infusion reaction, Hypokalemia, Hyponatremia, Hypoglycemic attack (3.7\%).

The Present study notify that Among 27 ADRs, 23(85.18\%) ADRs are found to be Non-serious and 04(14.81\%) ADRs are noticed as Serious, 04serious reactions are found to be Life Threatening, None of the reported reactions led to death, Out of 23 non-serious reactions 3 is found to be hospitalized. In this study, Majority of the ADRs20 (74.07\%) were cured within 1-5 days, whereas $3(11.11 \%)$ ADRs took less than one day to cure and $4(14.81 \%)$ ADRs took more than 5 days to cure. Various Causality Assessment Scales revealed the following data:

According to Naranjo Causality Assessment Scale, 24(88.88\%) reactions are found to be Possible and only 3(11.11\%) reactions are found to be probable. According to WHO-UMC Causality Assessment scale, 22(81.48\%) reactions are Probable/Likely and remaining 05(18.51\%) reactions are possible. According to Hartwig Severity Assessment Scale, 07(25.92\%) ADRs are found to be mild, 17(62.96\%) ADRs are found to be moderate, 3(11.11\%) ADRs are found to be Severe.

This study shows that $17(62.96 \%)$ ADRs are recovered, 02(7.4\%) ADRs are Unrecovered, $02(7.4 \%)$ are remained as Continuing, $04(14.81 \%)$ are Recurrent of symptoms,01(3.7\%) are fatal,01(3.7\%) are Unknown.

In this study, 19(70.37\%) ADRs were either managed by with drawl of the suspected Drug, 05(18.51\%) ADRs by Substituted another Drug,03(11.11\%) ADRs were reduced Dose. The duration of study was of short period and patients were randomly involved into the study. Hence the sample size was less, which is a major limitation of our study.

\section{CONCLUSION}

Pharmacovigilance is concerned with Identifying and Reporting of Adverse Drug Reaction associated with Medication 
with Minimizing Hazardous Which Harms the Patient. At the study site there is an under reporting of Adverse Drug Reaction. Clinical Pharmacist in a Health Care Team Plays a vital role in usage of Medications appropriately and safely which in turn reduces Drug related problems. Systematic Approach toward Adverse Drug Reaction Identifying and Reporting will Help health care professionals in reducing Adverse Drug Reaction.

\section{Acknowledgement: None}

\section{Conflict of Interest: None}

\section{Source of Funding: None}

\section{Ethical Approval: Approved}

\section{REFERENCES}

1. Muhammad UK, Akram A.The Impact of Clinical Pharmacists ${ }^{\text {ee }}$ Interventions on Drug Related Problems in a Teaching Based Hospital. International Journal of Pharmaceutical and Clinical Research. 2014; 6(3): 276-280.

2. Nimmy NJ, Aneesh PM, Narmadha MP, Udupi RH, Binu KM. A Survey on the Prevalence of Thyroid Disorder Induced by Demography and Food Habits in South Indian Population. Indian Journal of Pharmacy Practice .2012;5(2); 40-52.

3. Celin AT, Seuma J, Ramesh A. Assessment of Drug Related Problems in Stroke Patients Admitted to a South Indian Tertiary Care Teaching Hospital Indian Journal of Pharmacy Practice.2012 ;5(4); 28-33.

4. Jimmy OD, Shobha Rani RH,Indira R, Ramjan S.Study of Drug-drug Interactions in the Medication Charts in Medicine Wards at a Tertiary Care Hospital, Bangalore Indian Journal of Pharmacy Practice, India. 2012; 5(4); 61-64.

5. Abubakar S,Githa K, Kiran N, Sreebala Indian Journal of Pharmacy Practice, A Study on Pattern of Poisoning Cases in a Tertiary Care Hospital, Bangalore.2012; 7(1); 13-17.

6. Anuj KP,Subodh K ,Manish k,Lalit m, Harihar d. Study of Drug Utilization Pattern for Skin Diseases in Dermatology OPD of an Indian Tertiary Care Hospital - A
Prescription Survey Journal of Clinical and Diagnostic Research. 2016;10(2): 1-6

7. Vineeta D Sharad P, Ganachari MS, Geetanjali S and Santosh S Assessment of Drug Prescribing Pattern and Cost Analysis for SkinDisease in Dermatological Department of Tertiary Care Hospital: An Interventional Study India Journal of Pharmacovigilance 2016; 4(3);01-06

8. Leya P Babu Johnson V Babu, Shaji George, Nimmy Robin, Joicy Jose.Adverse Drug Reactions in Drug-Resistant Tuberculosis Management: A Clinical Approach Indian Journal of Pharmacy Practice, INDIA. 2021;14(2); 73-88

9. Thennarasu Palani Ramakrishnan V Trichur, Selina Grace Kurian, Selvabharathi M, Sivashankari M, Shalini AS, Srikanth Jeyabalan, Assessment of General Characteristics of Geriatric Patients Admitted in a Tertiary Care Teaching Hospital INDIA 2021;14(2);89-95

10. Konda Nikhitha,Sikilambatla SK, Meraboina P, Maram CE.Assessment of Quality of Life in Diabetes and Hypertensive Patients Attending Tertiary Care Hospitals in Khammam Region Indian Journal of Pharmacy Practice, INDIA 2021;14(2);96-101

11. Rekha B, Arvind D, Pankaj D, Dinesh KM. An Epidemiological Observation of Judicious use of Antibiotics in Dhar District, Indian Journal of Pharmacy Practice INDIA 2021;14(2);102-105

12. Vivek R, Priti D. Effect of Remedial Measures on Incidence and Awareness about Medication Errors in an Indian Tertiary Care Hospital, Indian Journal of Pharmacy Practice INDIA. 2021;14(2);106113.

13. Priti P. D, Akshay VM, Akshay PC. Retrospective Analysis of Look-alike and Sound-alike Drug Incidents in a Tertiary Care Hospital, Indian Journal of Pharmacy Practice INDIA 2021;14(2);114-117.

14. Vishnudas KS, Swetha S.Sheba SC, Angel S, Silsha P1, Arumukhan S, Ganapathi B. Assessment of Guideline Adherence and Hypertension Control in a Tertiary Care Hospital in Calicut, Indian Journal of Pharmacy Practice, India. 2021;14(2);140144.

15. Haroledpeter P.L.Sampathkumar M, Thirupathykumaresan P,Keerthiyurenikka J, Murugakadavul A, Rajasmith PM.A 
Prospective Study of Antibiotic Prescribing Pattern among Pediatrics in a Government District Headquarters Hospital, South India, Indian Journal of Pharmacy Practice, INDIA 2021;14(2);123-127.

16. Ranjeth R Amritha M.S1, Resmi V I. John W. A Prospective Comparative Study of Tacrolimus and Corticosteroids in the Treatment of Atopic Dermatitis, Indian Journal of Pharmacy Practice INDIA 2021 ;14(2),118-122.

17. Flegel, Ken. "Tertiary hospitals must provide general care.” CMAJ : Canadian Medical Association journal $=$ journal de l'Association medicale canadienne vol. 187,4 (2015): 235. doi:10.1503/cmaj.150056

18. Akhila D, Karthika N, Manasa B, Rajareddy R, Satyavardhan RN.Pharmacogenomics and Adverse Drug Reactions: Carbamazepine Induced Steven Johnsons Syndrome, Indian Journal of Pharmacy Practice INDIA. 2021;14(2),145-147.

19. Khaled M,Yasameen MM, Lyna I, Abdulaleem M.A Case of ThymomaMyasthenia Gravis in an Adult Malaysian Patient with Anterior Mediastinal Lesion, Indian Journal of Pharmacy Practice .2021;14(2),148-150.

20. Shirisha, Satish C, Shravani K, Venkateshwarlu E.Cefotaxime Induced Staphylococcal Scalded Skin Syndrome: A Case Report,Indian Journal of Pharmacy Practice INDIA,2021;14(2),151-153.

21. Arathi Thekke, Tonya CT, Gopika KS, Maneesha T, Kabeer P K.Case Report on Chronic Actinic Dermatitis,Indian Journal of Pharmacy Practice INDIA; 2021, 14(2),154-156.

22. Ramesh G,Aikaterini L.Impacts of COVID19 on Pharmacy Studies,Indian Journal of Pharmacy Practice ;2021,14(2),157-160.

23. Venkaraddi M C, Allu H, Prasanna k, Hemanth N, Chandrakanth M. Adverse Drug Reaction Monitoring and Reporting at H.S.K Hospital and Research CenterBagalkot. Indian Journal of Pharmacy Practice. 2016; 9(1): 49.
24. Palanisamy S, Arul Kumaran KSG, Rajasekaran A. A Prospective Study on Adverse Drug Reaction in tertiary care south Indian Hospital. Indian Journal of Pharmacy Practice. 2013; 6(2) :50.

25. Ponnusankar S, Megha G, Roopa BS, Raja. Pattern of adverse Drug Reaction in a Govt. District Headquarters Hospital in Tamil Nadu, India. Indian Journal of Pharmacy Practice.2016; 9(9): 33.

26. Dilip C, Lisa MM, Saraswathi R, Divya R, Adverse Drug Reaction monitoring in a tertiary level referral Hospital, Kerala. Indian Journal of Pharmacy Practice. 2012; 5(2):29.

27. Anita G, Anjleen K, Prashant S, Hema C. Adverse Drug Reaction Pattern in a Tertiary level teaching Hospital: A Retrospective Study. Indian Journal of Pharmacy Practice. 2017; 10(1): 28-30.

28. Gor AP, Desai SV. Adverse Drug Reactions (ADR) in the in Patients of Medicine Department of a Rural Tertiary Care Teaching Hospital and Influence of Pharmacovigilance in Reporting ADR. Indian Journal of Pharmacy Practice. 2008;40(1): 37- 40.

29. Rehan HS, Vasudev.K, Tripathi CD. Adverse drug reaction monitoring: Knowledge, attitude and practices of medical students and prescribers. Med $\mathrm{J}$ India. 2002 Jan -Feb; 15(1): 24.

30. Rohit SR. Evaluation of Potentially Inappropriate Medication Use and Risk of Adverse Drug Reactions in Hospitalized Older Adults: An Observational Study in a Tertiary Care Hospital. Indian Journal of Pharmacy Practice 2018 June; 11(2): 79.

How to cite this article: Mohsina F.P, Faheem I.P, Aquil-ur-Rahim Siddiuque et.al. A study on pharmacist mediated intervention in identifying and reporting of adverse drug reactions at a tertiary care hospital. Gal Int J Health Sci Res. 2021; 6(4): 26-41. DOI: https://doi.org/10. 52403/gijhsr.20211006 